\title{
Stable observation of additive superpositions of of Partial Differential Equations
}

\author{
Enrique Zuazua ${ }^{\mathrm{a}, \mathrm{b}}$ \\ ${ }^{a}$ BCAM - Basque Center for Applied Mathematics, Alameda Mazarredo 14, E-48009 Bilbao, Basque Country, Spain \\ ${ }^{\mathrm{b}}$ Ikerbasque - Basque Foundation for Science,48009, Bilbao, Basque Country, Spain
}

\begin{abstract}
We address the problem of observing the solutions of a given Partial Differential Equations (PDE), submitted to unknown perturbations. The problem under consideration is motivated by the issue of averaged control and observation. In the latter, one aims at determining the initial datum of a dynamics, submitted to unknown perturbations, by means of measurements done on the average of solutions with respect to the unknown parameters.

We do it in two different cases.

First, we assume that a given dynamics is perturbed in an additive manner by a state governed by a PDE, but not necessarily of the same type. In particular we consider the additive superposition of multi-physics solutions of wave and heat type, for instance.

Assuming the PDEs under consideration have constant coefficients, we prove that, the by now well-known observability properties of PDE (mainly heat and wave equations) from open subsets of the domain where the dynamics evolves, are stable under these additive perturbations.

The proof is rather systematic and easy to apply. It consists in composing all but one (the observable one) PDE operators so to reduce the problem to the consideration of a single equation and applying its known observability properties. The same proof leads to very interesting open problems for systems involving PDEs with variable coefficients where commutators lead to non-local lower order perturbations.

We then consider a particular case of a PDE depending on a parameter in a continuous manner. We show that the average is governed by the superposition of two distinct solutions, corresponding to the extremal values of the parameter, and experiencing an extra regularizing effect. This allows showing that the averaged observability property holds, for the continuous average, but to the prize of loosing a finite number of derivatives. This opens a wide range of interesting problems to be explored.
\end{abstract}

Email address: zuazua@bcamath.org (Enrique Zuazua).

Preprint submitted to Elsevier Science

June 29, 2015

(C) 2016. This manuscript version is made available under the Elsevier user license http://www.elsevier.com/open-access/userlicense/1.0/ 


\section{Introduction}

Motivated by the problem of averaged control and observation, in this paper we consider various situations in which a given Partial Differential Equation (PDE) is submitted to unknown perturbations.

The problem of averaged control and observation was formulated in [20] both for finite and infinitedimensional systems. The issue under consideration, in the context of control, can be formulated as follows. Given a system for which the parameters entering on it are not completely known, one aims at choosing a control that performs well at the level of controllability, i. e. with the goal of steering the system to a given final configuration. But the control has to be chosen independently of the unknown parameters. The issue of averaged control then makes sense. It consists on choosing the control so that the average of the states with respect to the unknown parameter is controlled.

Averaged controllability is equivalent to averaged observability. In the latter the issue is to determine the initial datum of a system in terms of the averages of partial measurements done on the solutions with respect to the unknown parameters.

In [20] we gave a complete characterization of that property for linear autonomous finite dimensional systems in terms of an averaged rank condition. In [14] that issue was addressed in the context of wave equations by dealing with systems of finitely many wave equations with different velocities of propagation.

In the present paper we further analyze this issue in the context of PDE. We do it in two different situations, both leading to surprising results.

First we consider the problem of determining the initial datum leading to the dynamics from partial observations made on an open subset of the domain where the dynamics evolves. But the dynamics is submitted to unknown additive perturbations. We do it in the context of PDEs with constant coefficients, assuming that the additive perturbation obeys another PDE, not necessarily of the same type as the one governing the observed dynamics.

This property is related but stronger than the one of averaged observability mentioned above. Indeed, in the context of averaged observability, one deals with averages of solutions of parameter dependent dynamics, but all departing from the same initial data. But here the additive perturbations can be arbitrary, departing from any initial source and governed by an arbitrary dynamics, the only restriction being to belong to the class of solutions of another PDE.

To fix ideas and illustrate the nature of the results we obtain, we first consider the heat dynamics, perturbed by an additive unknown solution of a wave equation. As we shall see, the observability of the heat equation from an arbitrary open subset of the domain where the system evolves, in an arbitrarily short time, ensures that the same property is preserved when the heat observation is perturbed by the additive wave signal, regardless what its shape or original source is. Our proof is rather systematic and consists in applying the wave operator to the superposition of both heat and wave solutions, so to reduce the problem to the heat dynamics.

The same technique can also be applied all the way around. Assuming that the observation region and time satisfy the so-called Geometric Control Condition (GCC) ([4]), we show that the wave component can be observed robustly when adding unknown heat perturbations.

More generally, the proof applies for systems of PDEs involving finitely many PDEs with constant coefficients. As a particular case, it can also be applied when all of them are of the same type, but not necessarily starting from the same initial datum as assumed in the context of averaged observation. Thus, in particular, the results apply to systems of wave equations and heat equations, with constant coefficients. But, when applied to systems of equations with variable coefficients, our proof yields equations involving non-local lower order commutators whose absorption poses challenging problems.

By duality, these robust observability results lead to new controllability properties.

This method does not suffice to analyze systems involving infinitely-many equations, or equations in- 
volving perturbations with respect to a continuous parameter. We thus also consider a simple situation in which the evolution equation under consideration depends continuously on a multiplicative unknown parameter. We give an explicit representation formula for the continuous average showing that it is governed by the average of two solutions corresponding to the two extremal values of the parameter, showing a phenomenon of gain of regularity. This allows extending the existing results of averaged observability of finitely many PDE to these continuous averages, to the prize of loosing a finite number of derivatives in the (Sobolev) norm observed. This issue has been systematically analyzed in [17].

The rest of the paper is organized as follows. In the first section we consider several situations in which the stability of the observability inequalities is proved by additive superposition of different dynamics. First, in Section 2 we consider the problem of the observation of the heat equation, submitted to an additive unknown wave-like perturbation. We then consider the reverse problem in which the wave dynamics is perturbed by a heat like one. In Section 3 the case of continuous averages is analysed. In Section 4 we present some open problems and perspectives for future research.

\section{Additive superposition of different dynamics}

\subsection{Observation of the heat equation under unknown additive wave perturbations}

Consider the following system, involving only two modes, whose solutions, $u_{1}, u_{2}$, satisfy respectively a heat and a wave equation:

$$
\begin{aligned}
u_{1, t}-\Delta u_{1} & =0, & & (t, \mathbf{x}) \in[0, T] \times \Omega \\
u_{2, t t}-\Delta u_{2} & =0, & & (t, \mathbf{x}) \in[0, T] \times \Omega \\
u_{i} & =0, & & (t, \mathbf{x}) \in[0, T] \times \partial \Omega, \quad i=1,2 \\
u_{1}(0, \cdot) & =\varphi(\cdot), & & \mathbf{x} \in \Omega .
\end{aligned}
$$

We assume that the main dynamics, the one we want to observe, is given by $u_{1}$, governed by the heat equation.

The wave solution, $u_{2}$, is adding an unknown additive perturbation. Note that nothing is known about the initial data of $u_{2}$. We only know that $u_{2}$ solves the wave equation.

We are trying to recover the whole solution of the heat equation or, equivalently, its initial datum $\varphi$, out of partial measurements of the superposition $u_{1}+u_{2}$ taken on a non-empty open subset $\omega$ of the domain $\Omega$ where the system evolves, that is assumed to be a bounded open set of $\mathbf{R}^{n}, n \geq 1$, during a time interval $T>0$.

Identifying the initial datum of a given PDE in terms of partial measurements is a classical control problem, due to the duality and equivalence between observability and controllability (see [19]), but also in the theory of inverse problems (see [6]).

The novelty of the problem we address here is that the observation estimates hold in the presence of additive unknown perturbations, under the sole assumption that they fulfill another PDE with constant coefficients.

Our main result concerning the system above is as follows:

Theorem 2.1 Let us assume that $\omega$ is an open non-empty subset subset of $\Omega$ and $T>0$ be arbitrary.

Then, there exists a constant $C$ such that the following estimate holds

$$
\left\|u_{1}(T)\right\|_{L^{2}(\Omega)}^{2} \leq C \int_{0}^{T} \int_{\omega}\left|u_{1}+u_{2}\right|^{2} d \mathbf{x} d t,
$$

for all solutions $\left(u_{1}, u_{2}\right)$ of the above system. 
Remark 1 Several remarks are in order.

- As observed in [11], in the absence of perturbation $u_{2}$, one can even achieve a slightly stronger observability property of the heat equation. Indeed, when expanding the solution of the heat equation in terms of the Fourier coefficients $\hat{\varphi}_{k}$ of the initial datum $\varphi$, on the basis of eigenfunctions of the Laplacian, one gets

$$
\sum_{k} e^{-c \sqrt{\lambda_{k}}}\left|\hat{\varphi}_{k}\right|^{2} \leq C \int_{0}^{T} \int_{\omega}\left|u_{1}\right|^{2} d \mathbf{x} d t
$$

for some $c, C>0$. This inequality also holds in the context above so that, for suitable constants $C, c>0$, it holds

$$
\sum_{k} e^{-c \sqrt{\lambda_{k}}}\left|\hat{\varphi}_{k}\right|^{2} \leq C \int_{0}^{T} \int_{\omega}\left|u_{1}+u_{2}\right|^{2} d \mathbf{x} d t .
$$

This improves the estimate in (2) where the observed norm of $u_{1}(T)$ involves rather weights of the order of $\exp \left(-c \lambda_{k} T\right)$, because of the regularizing and dissipative effect of the heat equation.

- Surprisingly enough, the inequality we obtain is independent of the additive perturbation of the solution $u_{2}$ of the wave equation under consideration, and in particular of its initial data, which do not need to be related to the heat component $\varphi$. This means that the classical observability inequality for the heat equation is preserved, regardless what the wave-like additive unknown perturbation is. The estimates we obtain depend also continuously on the constant coefficients entering in the wave equation. Here, to simplify the presentation, we have taken the d'Alembert operator with constants normalized to one. But a similar result holds for a general wave equation with constant coefficients, involving possibly lower order terms.

- We have stated the result in the case where $u_{2}$ solves the wave equation but, in fact, we could also consider solutions $u_{2}$ of other constant coefficient equations, as for instance plate equations, or other heat equations.

- The case of systems of heat equations will be addressed later. In this way we will extend the result in [14] obtained using transmutation and the averaged observability of systems of wave equations, requiring $\omega$ to fulfil the GCC. Our new result applies to any observation open subset $\omega$.

- The idea of applying some of the PDE operators entering in the system to annihilate some of the other components of the system has been employed in previous articles (see, for instance, [1] for systems of heat equations). But there the goal was different, aiming to observe all the components of the system out of observations done only on some of them. By duality, that situation corresponds to the control of the whole system by means of a reduced number of controls and not to the additive perturbation one we prove here.

- For the sake of simplicity we have described here the case of systems involving two components. But the same arguments apply for systems involving finitely-many equations. The key is the fact that all PDE operators commute, and that the equation governing the main dynamics satisfies the observability inequality.

Proof. We denote by $P_{1}$ the heat operator, $P_{1}=\partial_{t}-\Delta$, and by $P_{2}$ the wave one, $P_{2}=\partial_{t t}-\Delta$. We introduce the new variable

$$
v_{1}=P_{2}\left(u_{1}+u_{2}\right)=P_{2} u_{1} .
$$

Note that in this new variable, that is obtained by applying the wave operator to the superposition of $u_{1}$ and $u_{2}$, the contribution of the wave component $u_{2}$ vanishes since it is precisely a solution of the wave equation, involving the operator $P_{2}$.

In fact $v_{1}$ solves the heat equation with the same Dirichlet boundary conditions: 


$$
\begin{array}{rlrl}
v_{1, t}-\Delta v_{1} & =0, & (t, \mathbf{x}) & \in[0, T] \times \Omega \\
v_{1}=0, & (t, \mathbf{x}) & \in[0, T] \times \partial \Omega .
\end{array}
$$

We now recall the following classical observability inequality for the heat equation ([9]) ensuring that, there exists a constant $C>0$ such that

$$
\left\|v_{1}(T)\right\|_{L^{2}(\Omega)}^{2} \leq C \int_{0}^{T} \int_{\omega}\left|v_{1}\right|^{2} d x d t .
$$

In fact, using the regularizing effect of the heat equation, the same result can be proved when observations are made in Sobolev spaces of negative order. Namely, for any $s>0$ there exists $C_{s}>0$ such that

$$
\left\|v_{1}(T)\right\|_{L^{2}(\Omega)}^{2} \leq C_{s}\left\|v_{1}\right\|_{H^{-s}(\omega \times(0, T))}^{2} .
$$

As observed in [11] and [9], and mentioned above, this can be rewritten as

$$
\int_{\omega \times(0, T)} e^{-A / t} v_{1}^{2} d x d t \leq C_{s}\left\|v_{1}\right\|_{H^{-s}(\omega \times(0, T))}^{2},
$$

or

$$
\sum_{k} e^{-c \sqrt{\lambda_{k}}}\left|\hat{v}_{1 k}(0)\right|^{2} \leq C_{s}\left\|v_{1}\right\|_{H^{-s}(\omega \times(0, T))}^{2},
$$

$\hat{v}_{1 k}(0)$ being the Forier coefficients of $v_{1}(0)=\left(\Delta^{2}-\Delta\right) \varphi$, i. e. $\hat{v}_{1 k}(0)=\left(\lambda_{k}^{2}+\lambda_{k}\right) \hat{\varphi}_{k}$, thus yielding stronger observed norms on the Fourier coefficients $\varphi_{k}$ of $\varphi$. These inequalities are well-known to be sharp although the optimal constant $c$ entering in the exponential weights is often unknown $([9])$.

We now observe that

$$
\left\|v_{1}\right\|_{H^{-2}(\omega \times(0, T))}=\left\|P_{2}\left(u_{1}\right)\right\|_{H^{-2}(\omega \times(0, T))}=\left\|P_{2}\left(u_{1}+u_{2}\right)\right\|_{H^{-2}(\omega \times(0, T))} \leq C\left\|u_{1}+u_{2}\right\|_{L^{2}(\omega \times(0, T))} .
$$

Applying (10) with $s=2$ to the heat solution $v_{1}$ and combining it with (11) and employing the form of its Fourier coefficients we deduce that

$$
\sum_{k} e^{-c \sqrt{\lambda_{k}}} \lambda_{k}^{4}\left|\hat{\varphi}_{k}\right|^{2} \leq C|| v_{1} \|_{H^{-2}(\omega \times(0, T))}^{2} \leq C \int_{0}^{T} \int_{\omega}\left|u_{1}+u_{2}\right|^{2} d \mathbf{x} d t .
$$

Consequently we also have

$$
\sum_{k} e^{-c \sqrt{\lambda_{k}}}\left|\hat{\varphi}_{k}\right|^{2} \leq C \int_{0}^{T} \int_{\omega}\left|u_{1}+u_{2}\right|^{2} d \mathbf{x} d t
$$

as stated.

This, of course, also yields the weaker estimate (19) since the norm $\left\|u_{1}(T)\right\|_{L^{2}(\Omega)}^{2}$ involves rather the weaker weights $e^{-2 \lambda_{k} T}$ in the Fourier coefficients.

Remark 2 The proof above uses the fact that the two operators $P_{1}$ and $P_{2}$ commute. The fact that the coefficients of the PDE under consideration are constant is not essential. The same applies to heat equations of the form

$$
\partial_{t} u_{1}-\operatorname{div}\left(a(x) \nabla u_{1}\right)=0,
$$

for instance, with Lipschitz coefficients $a=a(x)$, provided that the second wave operator also involves the same elliptic operator, namely,

$$
\partial_{t t}^{2} u_{2}-\operatorname{div}\left(a(x) \nabla u_{2}\right)=0 .
$$

But, in the proof above, not only the operators need to commute but also the boundary conditions to be the same. 
In an abstract setting, the results above apply to systems of the form

$$
\partial_{t} u_{1}+A u_{1}=0 ; \quad \partial_{t t}^{2} u_{2}+A u_{2}=0,
$$

A being a strictly negative operator so that both equations above generate two continuous semigroups. This requires, of course, the underlying elliptic operators A to coincide but also the boundary conditions to be compatible.

The obtained result allows transferring the observability properties of the parabolic dynamics given by $u_{1}$, to the superposition of both $u_{1}$ and $u_{2}$.

The inspection of the proof above shows that the same technique applies to any other perturbative dynamics governing $u_{2}$, provided it only involves the operator A. One could consider a plate-like abstract model of the form

$$
\partial_{t t}^{2} u_{2}+\operatorname{div}\left(a(x)\left(\operatorname{div}\left(a(x) \nabla u_{2}\right)\right)\right)=0,
$$

but with compatible boundary conditions:

$$
u_{2}=\operatorname{div}\left(a(x) \nabla u_{2}\right)=0 \quad \text { on } \quad \partial \Omega \times(0, T),
$$

that, in an abstract frame, would correspond to:

$$
\partial_{t t}^{2} u_{2}+A^{2} u_{2}=0 .
$$

\subsection{Unknown heat perturbations of a wave process}

In this section we consider the same system but this time we aim to observe the wave component, assuming that the unknown additive perturbation is the one introduced by the heat equation. Thus, we consider the system

$$
\begin{array}{cl}
u_{1, t}-\Delta u_{1}=0, & (t, \mathbf{x}) \in[0, T] \times \Omega \\
u_{2, t t}-\Delta u_{2}=0, & (t, \mathbf{x}) \in[0, T] \times \Omega \\
u_{i}=0, \quad(t, \mathbf{x}) & \in[0, T] \times \partial \Omega, \quad i=1,2 \\
u_{2}(0, \cdot)=\varphi(\cdot), u_{2, t}(0, \cdot)=\psi(\cdot), \quad \mathbf{x} \in \Omega .
\end{array}
$$

We are going to show that the standard observability inequality for the wave equation is not affected by the additive perturbation that the heat process introduces. In this case no information will be used on the initial data of the heat solution.

This time we assume that $(\omega, T)$ satisfy the so-called GCC ([4]) so that the following observability inequality holds for the solutions of the wave equation:

$$
\|\varphi\|_{L^{2}(\Omega)}^{2}+\|\psi\|_{H^{-1}(\Omega)}^{2} \leq C \int_{0}^{T} \int_{\omega} u_{2}^{2} d x d t .
$$

The following holds:

Theorem 2.2 Let us assume that $(\omega, T)$ satisfy the $G C C$.

Then, there exists a constant $C$ such that the following estimate holds

$$
\|\varphi\|_{H^{2}(\Omega)}^{2}+\|\psi\|_{H^{1}(\Omega)}^{2} \leq C|| u_{1}+u_{2} \|_{H^{2}(\omega \times(0, T))}^{2},
$$

for all solutions $\left(u_{1}, u_{2}\right)$ of the above system.

Proof. The proof follows the same arguments as that of Theorem 2.1 but this time applying the heat operator $P_{1}$ to the sum $u_{1}+u_{2}$ and employing the observability inequality (15) of the wave equation applied to $v_{2}=P_{1} u_{2}$. 
Obviously, the $L^{2}(\omega \times(0, T))$-norm of $v_{2}$ can be bounded above by the $H^{2}(\omega \times(0, T))$-norm of $u_{1}+u_{2}$. On the other hand, one needs to identify the initial datum of $v_{2}=P_{1} u_{2}$. In particular, we have

$$
v_{2}(0)=u_{2, t}(0)-\Delta u_{2}(0)=\psi-\Delta \varphi,
$$

and

$$
v_{2, t}(0)=u_{2, t t}(0)-\Delta u_{2, t}(0)=\Delta u_{2}(0)-\Delta u_{2, t}(0)=\Delta(\varphi-\psi) .
$$

It is easy to see that an estimate of $\left(v_{2}(0), v_{2, t}(0)\right)$ in $L^{2}(\Omega) \times H^{-1}(\Omega)$ is equivalent to an estimate of $(\varphi, \psi)$ in $H^{2}(\Omega) \times H_{0}^{1}(\Omega)$.

This completes the proof.

Remark 3 The following comments are also worth to be taken into account:

- The observability of the wave equation also holds in Sobolev spaces of negative order. This can be easily proved, employing duality, by the arguments in [10] that allows building smooth controls for smooth data, starting up on the $L^{2}$-observability inequality that the GCC provides. Thus, the result above also holds in weaker spaces. In particular, it follows that:

$$
\|\varphi\|_{L^{2}(\Omega)}^{2}+\|\psi\|_{H^{-1}(\Omega)}^{2} \leq C \int_{0}^{T} \int_{\omega}\left|u_{1}+u_{2}\right|^{2} d x d t .
$$

- The same results hold for wave equations with variable coefficients, provided they are smooth enough, and the heat perturbation is generated by the same elliptic operator, with compatible boundary conditions.

At this respect it is important to note that, even in $1-d$ space dimension, the coefficients of the wave equation need to be in BV for observability to hold (see [5]). This is not the case for heat like equations where, in the particular $1-d$ case, bounded measurable coefficients suffice (see [2]). This shows an interesting difference in $1-d$. While the observability of the heat equation with bounded measurable coefficients remains under wave-like additive perturbations, one needs BV coefficients to preserve the wave like observability inequality.

- As we shall see below, this has also interesting consequences in what concerns Fourier series.

\subsection{Systems of heat equations}

In this section we apply the previous techniques to systems of constant coefficient heat equations.

We improve the results in [14] in two different ways. On one hand, we observe that the GCC imposed in [14], as one could expect, is unnecessary in the parabolic setting. On the other, we show that there is no necessity of correlating the initial data of the various heat components. Indeed, in [14], motivated by the problem of averaged observation, we assumed that all the heat components fulfilled the same initial data. Here, we obtain a stronger result and it rather concerns the scenario in which there is a leading heat process and all others are considered as being additive unknown perturbations.

For simplicity we state the result in the case of two equations:

$$
\begin{aligned}
& u_{1, t}-\Delta u_{1}=0, \quad(t, \mathbf{x}) \in[0, T] \times \Omega \\
& u_{2, t}-c_{2} \Delta u_{2}=0, \quad(t, \mathbf{x}) \in[0, T] \times \Omega \\
& u_{i}=0, \quad(t, \mathbf{x}) \in[0, T] \times \partial \Omega, \quad i=1,2 \\
& u_{1}(0, \cdot)=\varphi \in L^{2}(\Omega) .
\end{aligned}
$$

We underline that no information is provided on the initial datum of $u_{2}$.

The following holds. 
Theorem 2.3 Let us assume that $\omega$ is an open non-empty subset subset of $\Omega, c_{2}>0, c_{2} \neq 1$ and $T>0$ be arbitrary.

Then, there exists a constant $C$ such that the following estimate holds

$$
\left\|u_{1}(T)\right\|_{L^{2}(\Omega)}^{2} \leq C \int_{0}^{T} \int_{\omega}\left|u_{1}+u_{2}\right|^{2} d \mathbf{x} d t
$$

for all solutions $\left(u_{1}, u_{2}\right)$ of the above system.

Proof. The proof follows the same arguments as before but this time with the heat operators $P_{1}=\partial_{t}-\Delta$ and $P_{2}=\partial_{t}-c_{2} \Delta$. Applying the heat operator $P_{2}$ to the sum $u_{1}+u_{2}$ and employing the observability inequality of the heat equation $P_{1}$ applied to $v_{1}=P_{2} u_{1}$.

Remark 4 - As in the context of Theorem 2.1, the estimate (19) can be improved to get an estimate of the form (4), which significantly improves the exponential weights on the observed quantity.

- The same result holds for a system of finitely many heat equations, provided the diffusivity of all of them differs from the fist one.

- The same result holds as well for systems of heat equations, provided all of them are generated by the same elliptic operator, possibly with variable coefficients but, of course, with the same boundary conditions.

\subsection{Ingham like inequalities}

The proof we have applied here is inspired in the techniques we employed in [8] to deal with wave equations on graphs. There the time-periodicity of solutions of the $1-d$ equation was used to annihilate some of the components of the system under consideration.

These techniques, applied in the hyperbolic-parabolic setting of this paper, have interesting and nontrivial consequences that we briefly discuss here.

Consider two non-harmonic Fourier series, representing the values at a given point of the solutions of the $1-d$ wave and heat equations, respectively:

$$
u_{h}(t)=\sum_{k} a_{k} e^{-k^{2} t}, \quad u_{w}(t)=\sum_{k} b_{k} e^{i k t},
$$

and their superposition

$$
u(t)=u_{h}(t)+u_{w}(t)=\sum_{k}\left[a_{k} e^{-k^{2} t}+b_{k} e^{i k t}\right]
$$

The following holds:

Theorem 2.4 For all $T \geq 2$ there exist positive constants $c=c(T), C>0$ such that

$$
\sum_{k} e^{-c(T) k^{2}}\left|a_{k}\right|^{2} \leq C \int_{0}^{T}\left|u_{h}(t)+u_{w}(t)\right|^{2} d t,
$$

for all pairs of fails of coefficients $\left\{a_{k}\right\}$ and $\left\{b_{k}\right\}$.

Note that this generalizes a well-known estimate for series of real exponentials, but under the perturbation of additive complex exponentials, when $T>2$, and with constants that are totally independent of the perturbation introduced by the wave-like coefficients $\left\{b_{k}\right\}$.

Proof. The proof is very simple. It suffices to observe that $u_{w}$ is time-periodic of period 2 . Then

$$
u(t+2)-u(t)=u_{h}(t+2)-u_{h}(t)=\sum_{k}\left[\left(e^{-2 k^{2}}-1\right) a_{k} e^{-k^{2} t}\right] .
$$


Applying well-known inequalities for series of real exponentials ([16]) we get that

$$
\sum_{k}\left|e^{-2 k^{2}}-1\right|^{2} a_{k}^{2} e^{-(T-2) k^{2}} \leq C \int_{0}^{T-2}|u(t+2)-u(t)|^{2} d t \leq C \int_{0}^{T} u^{2}(t) d t,
$$

and this easily leads to the result.

Note that, the most striking aspect of the estimate is that, it is totally independent of the Fourier coefficients $\left\{b_{k}\right\}$ governing the wave-like perturbation. It seems that this surprising stability property of these classical inequalities for non-harmonic Fourier series was not noticed before. It would be interesting to make a more systematic analysis of these issues for the interaction of more general wave and heat spectra. We refer to [7] for more general results on this kind arising in the analysis of $1 d$ linearised compressible Navier-Stokes equations.

\subsection{Control consequences}

Observation and controllability properties are often closely linked through duality arguments (see [19]). Here we briefly present a control theoretical consequence of the observation results above.

We are going to derive a result of averaged control, which refers to the property of controllability of the average of various solutions of a parameter dependent system, by means of a single control which acts on each of them (see [20]).

Let us consider the controlled system of heat and wave equations:

$$
\begin{aligned}
y_{t}-\Delta y & =f \chi_{\omega}, \quad(t, \mathbf{x}) \in[0, T] \times \Omega \\
z_{t t}-\Delta z & =f \chi_{\omega}, \quad(t, \mathbf{x}) \in[0, T] \times \Omega \\
y=z & =0, \quad(t, \mathbf{x}) \in[0, T] \times \partial \Omega, \\
y(0, \cdot)=y^{0} & \in L^{2}(\Omega), \\
z(0, \cdot)=z^{0} \in L^{2}(\Omega), & z_{t}(0)=z^{1} \in H^{-1}(\Omega) .
\end{aligned}
$$

Here we do not make any geometric assumption on $\omega$, other than being open and non-empty. We are thus looking for an extension of the classical controllability property of the heat equation (see [12]) and this has to be achieved as a consequence of the observability inequality in Theorem 2.1. Note that the applied control $f$ is the same both in the heat and in the wave equation.

Consider the adjoint system:

$$
\begin{aligned}
p_{t}+\Delta p=0, & (t, \mathbf{x}) \in[0, T] \times \Omega \\
q_{t t}-\Delta q=0, & (t, \mathbf{x}) \in[0, T] \times \Omega \\
p=q=0, & (t, \mathbf{x}) \in[0, T] \times \partial \Omega, \\
p(T, \cdot)=p^{0}, & \\
q(T, \cdot)=q^{0}, q_{t}(T)=q^{1} . &
\end{aligned}
$$

We assume that the data at time $t=T$ of the wave component of adjoint solution $q$ are related to those of the heat component $p^{0}$ through linear operators so that the observation of the norm

$$
\sum_{k} e^{-c \sqrt{\lambda_{k}}}\left|\hat{p}_{k}^{0}\right|^{2}
$$

with $c>0$ is as in (4), ensures also the observation of the norm of $\left(q^{0}, q^{1}\right)$ in $L^{2}(\Omega) \times H^{-1}(\Omega)$.

Thus, we take initial data in the cone

$$
X_{M}=\left\{\left(p^{0}, q^{0}, q^{1}\right): \sum_{k} e^{-c \sqrt{\lambda_{k}}}\left|\hat{p}_{k}^{0}\right|^{2}<\infty ;\left\|q^{0}\right\|_{L^{2}(\Omega)}^{2}+\left\|q^{1}\right\|_{H^{-1}(\Omega)}^{2} \leq M \sum_{k} e^{-c \sqrt{\lambda_{k}}}\left|\hat{p}_{k}^{0}\right|^{2}\right\},
$$


for some $M>0$ and, more precisely, in a linear subspace of the form

$$
L_{\epsilon, \delta}=\left\{\left(p^{0}, q^{0}, q^{1}\right): \sum_{k} e^{-c \sqrt{\lambda_{k}}}\left|\hat{p}_{k}^{0}\right|^{2}<\infty ; \hat{q}_{k}^{0}=\varepsilon_{k} \hat{p}_{k}^{0}, \hat{q}_{k}^{1}=\delta_{k} \hat{p}_{k}^{0}\right\} .
$$

This can be done, for instance, by taking linear weighted relations: $\hat{q}_{k}^{0}=\varepsilon_{k} \hat{p}_{k}^{0}$ and $\hat{q}_{k}^{1}=\delta_{k} \hat{p}_{k}^{0}$, with $\left\{\varepsilon_{k}\right\}$ and $\left\{\delta_{k}\right\}$ decaying exponentially. We denote these smoothing operators respectively by $S_{\varepsilon}$ and $S_{\delta}$.

Obviously, under a suitable decay condition on $\left\{\varepsilon_{k}\right\}$ and $\left\{\delta_{k}\right\}$, the subspace $L_{\epsilon, \delta}$ is embedded into a cone of the form $K_{M}$ with a suitable $M>0$.

Then, both within $L_{\epsilon, \delta}$ and $K_{M}$, the observability inequality of Theorem 2.1

$$
\sum_{k}\left|\hat{p}_{k}^{0}\right|^{2} e^{-c \sqrt{\lambda_{k}}} \leq C \int_{0}^{T} \int_{\omega}|p+q|^{2} d x d t
$$

ensures also

$$
\sum_{k}\left|\hat{p}_{k}^{0}\right|^{2} e^{-c \sqrt{\lambda_{k}}}+\left\|q^{0}\right\|_{L^{2}(\Omega)}^{2}+\left\|q^{1}\right\|_{H^{-1}(\Omega)}^{2} \leq C \int_{0}^{T} \int_{\omega}|p+q|^{2} d x d t .
$$

We now consider the following quadratic functional in the class of solutions of the adjoint system above with data in $L_{\epsilon, \delta}$,

$$
J(p, q)=\int_{0}^{T} \int_{\omega}|p+q|^{2} d x d t+\int_{\Omega} y_{0} p(0) d x+\int_{\Omega} z^{1} q(0) d x-\int_{\Omega} z^{0} q_{t}(0) d x .
$$

According to $(27)$ the functional is coercive and achieves its minimum at $(\bar{p}, \bar{q})$. Taking the EulerLagrange equations at the minimizer one can see that, by means of the same control $f=\bar{p}+\bar{q}$ applied to both components, heat and wave, the solution $\left(y, z, z_{t}\right)$ can be controlled in the following averaged sense:

$$
y(T)-S_{\delta} z(T)+S_{\varepsilon} z_{t}(T)=0 .
$$

Note that there is no constraint on the data $\left(y^{0}, z^{0}, z^{1}\right)$ to be controlled. Thus, this is an extension of the classical null-controllability result for the solutions of the heat equation ensuring that $y(T)$ can be controlled to zero or, more generally, to any element of the set of reachable states that contains, in particular, the image of the heat semigroup at time $T$.

In the present case the heat trajectory is controlled to a suitable linear combination of both components of the wave trajectory. Note however that, in view of the regularizing effect of the heat equation, it is natural that this can only be done when the wave component is submitted to the regularizing effect of the operators $S_{\varepsilon}$ and $S_{\delta}$.

Minimizing the functional $J$ above on the cone $X_{M}$ one can also recover the controllability of the state $\left(y(T), z(T), z_{t}(T)\right)$ to the dual or polar cone.

But the observability result we have is stronger since the observability inequality holds, regardless what are the initial data $\left(q^{0}, q^{1}\right)$ of the wave component solution. The control properties above, thus, do not completely reflect the full power of the observability result we have obtained.

\section{Continuous averages}

The results of the previous section can be applied to the additive superposition of finitely many solutions of PDEs. On the other hand, as mentioned in the introduction, in the context of finite-dimensional systems, one can also handle the case where the unknown parameter varies continuously. It is then natural to consider the same problem at the PDE level. 
In this section we first consider the case of a heat equation for which the constant diffusivity is unknown within an interval of positive numbers. We show that the averages of these solutions can be rewritten as the average of two solutions corresponding to the extremal values of the diffusivity coefficient. This allows applying the results in section 2.3 to obtain the averaged observability and, as a consequence, the averaged controllability.

Once this is done we shall observe that the same principle can be applied for families of wave equations or even for general abstract systems. This technique, as it will be presented here, only applies to multiplicative perturbations of a given generator of the dynamics. It raises many interesting questions about the possible extensions to a more general setting.

This issue has been further developed in [17] where other models are analysed and averages are computed with respect to various density functions.

\subsection{Continuous averages of heat equations}

Let us consider the heat equation, depending on a diffusivity coefficient $\sigma$ ranging on a bounded interval $0<\sigma_{1} \leq \sigma \leq \sigma_{2}<\infty$ :

$$
\begin{aligned}
u_{t}-\sigma \Delta u & =0, \quad(t, \mathbf{x}) \in \mathbf{R}^{+} \times \Omega \\
u & =0, \quad(t, \mathbf{x}) \in \mathbf{R}^{+} \times \partial \Omega, \\
u(0, \cdot)=u^{0} & \in L^{2}(\Omega) .
\end{aligned}
$$

We are interested in the problem of averaged observability. Once again, given an open non-empty subset $\omega$ of $\Omega$, and $T>0$, we analyze to which extent the initial datum $u^{0}$ can be recovered by an observation over the set $\omega \times(0, T)$ of the average of the solutions with respect to $\sigma$. In other words, we define

$$
v(x, t)=\int_{\sigma_{1}}^{\sigma_{2}} u(x, t ; \sigma) d \sigma,
$$

where $u=u(x, t, \sigma)$ denotes the solution of the heat equation in (46).

As mentioned above, the main tool to prove observability inequalities for heat equations are the Carleman inequalities. They do not seem to be directly applicable to the continuous mean above since, in principle, of course, it is not governed by a heat equation. But a more careful analysis exhibits an interesting feature, according to which, the continuous average in (41) can be rewritten as the average of two particular solutions corresponding to the extremal diffusivities $\sigma_{1}$ and $\sigma_{2}$.

Indeed, note that

$$
U_{\sigma}(x, t)=u(x, t / \sigma ; \sigma),
$$

solves the same heat equation but with diffusivity $\sigma=1$. Then,

$$
u(x, t ; \sigma)=U(x, \sigma t)
$$

and therefore

$$
v(x, t)=\int_{\sigma_{1}}^{\sigma_{2}} u(x, t ; \sigma) d x=\int_{\sigma_{1}}^{\sigma_{2}} U(x, \sigma t) d \sigma=\frac{1}{t}\left[V\left(x, \sigma_{2} t\right)-V\left(x, \sigma_{1} t\right)\right],
$$

where

with $\xi(x)$ solution of

$$
V(x, t)=\int_{0}^{t} U(x, s) d s+\xi(x)
$$

$$
\begin{array}{rlrl}
-\Delta \xi & =u^{0}, \quad & \mathbf{x} \in \Omega \\
\xi & =0, \quad \mathbf{x} \in \partial \Omega
\end{array}
$$


which is a solution of

$$
\begin{aligned}
V_{t}-\Delta V & =0, \quad(t, \mathbf{x}) \in \mathbf{R}^{+} \times \Omega \\
V & =0, \quad(t, \mathbf{x}) \in \mathbf{R}^{+} \times \partial \Omega, \\
V(0, \cdot)=\xi & \in L^{2}(\Omega) .
\end{aligned}
$$

Thus, $t v(x, t)$ is a discrete average of two solutions of two heat equations with diffusivities $\sigma_{1}$ and $\sigma_{2}$, since each $W_{j}(x, t)=V\left(x, \sigma_{j} t\right)$ solves

$$
W_{j, t}-\sigma_{j} \Delta W_{j}=0 .
$$

Accordingly the results in section (2.3) apply so that

$$
\begin{aligned}
& \sum_{k} e^{-c \sqrt{\lambda_{k}}}\left|\hat{u}_{0, k}\right|^{2} \leq C \int_{0}^{T} \int_{\omega}\left|W_{1}-W_{2}\right|^{2} d \mathbf{x} d t=C \int_{0}^{T} \int_{\omega}|t v(x, t)|^{2} d \mathbf{x} d t \\
& =C\left\|t \int_{\sigma_{1}}^{\sigma_{2}} u(x, t ; \sigma) d \sigma\right\|_{L^{2}(\omega \times(0, T))}^{2} \leq C T^{2}\left\|\int_{\sigma_{1}}^{\sigma_{2}} u(x, t ; \sigma) d \sigma\right\|_{L^{2}(\omega \times(0, T))}^{2}
\end{aligned}
$$

In (34) $\hat{u}_{0, k}$ are the Fourier coefficients of the initial datum $u_{0}$. Note that, although the results in section (2.3) directly applied to $t v(x, t)$ would provide a similar estimate on the Fourier coefficients of $\xi$, the exponential estimate remains on $\hat{u}_{0, k}$, since there is an algebraic connection between the Fourier coefficients of $u_{0}$ and $\xi$ of the form

Accordingly, the following holds:

$$
\hat{\xi}_{k}=\hat{u}_{0, k} / \lambda_{k}
$$

Theorem 3.1 Let $\omega$ be an open non-empty subset of $\Omega$ and $T>0$. Assume the unknown diffusivity $\sigma$ of the system (46) ranges over the set $0<\sigma_{1} \leq \sigma \leq \sigma_{2}<\infty$. Then, there exists constant $C>0$ such that for every $u_{0}$ the averages of solutions of (46) with respect to the unknown diffusivity satisfy (34).

Remark 5 There is an important difference between the result obtained here and those in the previous section. In here all the heat equations are assumed to take the same initial datum. We thus have an averaged observability inequality. Note that in the previous section the initial data of the various heat equations could differ one from each other. But in that analysis only a finite number of equations was allowed while here we have a continuum of them.

This result has also interesting consequences in what concerns averaged control. The following holds.

Theorem 3.2 Let $\omega$ be an open non-empty subset of $\Omega$ and $T>0$. Assume the unknown diffusivity $\sigma$ of the controlled system

$$
\begin{aligned}
y_{t}-\sigma \Delta y & =f 1_{\omega}, \quad(t, \mathbf{x}) \in[0, T] \times \Omega \\
y & =0, \quad(t, \mathbf{x}) \in[0, T] \times \partial \Omega, \\
y(0, \cdot)=y^{0} & \in L^{2}(\Omega),
\end{aligned}
$$

ranges over the set $0<\sigma_{1} \leq \sigma \leq \sigma_{2}<\infty$. Then, for all $y^{0} \in L^{2}(\Omega)$ there exists a control $f \in L^{2}(\omega \times(0, T))$ such that the solutions $y=y(x, t ; \sigma)$ enjoy the property of averaged null controllability in the sense that

$$
\int_{\sigma_{1}}^{\sigma_{2}} y(x, T ; \sigma) d \sigma=0 .
$$

The proof of this result can be done by classical means using duality and the minimization of a suitable quadratic functional. In the present case the functional to be minimized among all possible solutions of (46) is

$$
J\left(\varphi^{0}\right)=\frac{1}{2} \int_{0}^{T} \int_{\omega}\left|\int_{\sigma_{1}}^{\sigma_{2}} \varphi(x, t ; \sigma) d \sigma\right|^{2} d x d t+\int_{\Omega} y^{0}(x) \int_{\sigma_{1}}^{\sigma_{2}} \varphi(x, 0, \sigma) d \sigma d x
$$


where $\varphi(x, t ; \sigma)$ stands for the solution of the adjoint system

$$
\begin{aligned}
-\varphi_{t}-\sigma \Delta \varphi & =0, \quad(t, \mathbf{x}) \in[0, T] \times \Omega \\
\varphi & =0, \quad(t, \mathbf{x}) \in[0, T] \times \partial \Omega, \\
\varphi(T, x, \sigma) & =\varphi^{0}(x) \in L^{2}(\Omega) .
\end{aligned}
$$

The average observability result above shows that for all $y^{0} \in L^{2}(\Omega)$ the functional $J$ is coercive in the Hilbert space constituted by the data $\varphi^{0}$ such that the corresponding solutions of the adjoint system are such that $\int_{\sigma_{1}}^{\sigma_{2}} \varphi(x, t ; \sigma) d \sigma$ belongs to $L^{2}(\omega \times(0, T))$. Then, the functional being continuous, convex and coercive in this Hilbert space $H$, it achieves its minimum at some $\tilde{\varphi}^{0}$ in $H$ and the needed control reads

$$
f(x, t)=\int_{\sigma_{1}}^{\sigma_{2}} \tilde{\varphi}(x, t ; \sigma) d \sigma .
$$

This is simply the implementation of the duality principle between averaged observability and control described in [20].

\subsection{An abstract setting}

In fact it is easy to see that the argument above applies in an abstract semigroup setting. It suffices to observe that the solutions of

$$
\begin{aligned}
& u_{t}+\sigma A u=0, \quad t \in \mathbf{R}^{+} \\
& u(0)=u^{0},
\end{aligned}
$$

where $A$ is the generator of a continuous semigroup in a Hilbert or Banach space, can be written, again, after multiplication by the time weight $t$ as the difference of two particular solutions corresponding to the extremal values $\sigma_{1}$ and $\sigma_{2}$, and a regularized initial datum $\xi=A^{-1} u_{0}$.

Then the tools of the previous section, about the superposition and additive perturbations of two commuting semigroups apply and yield averaged observability results. This can be done in the general abstract frame developed in [18].

In particular, the following holds:

Theorem 3.3 Let $A$ be the generator of a semigroup and $u(t ; \sigma)$ the corresponding solutions of (37).

Then, the average of solutions

$$
\int_{\sigma_{1}}^{\sigma_{2}} u(t, \sigma) d \sigma
$$

can be rewritten as

$$
\int_{\sigma_{1}}^{\sigma_{2}} u(t, \sigma) d \sigma=\frac{1}{t}\left[U_{2}(t)-U_{1}(t)\right]
$$

where $U_{1}$ and $U_{2}$ are, respectively, the solutions of system (37) with coefficients $\sigma_{2}$ and $\sigma_{1}$, respectively, and initial datum

$$
A^{-1} u^{0} \text {. }
$$

The proof of this identify follows exactly the same argument above on the heat equation. Obviously, in case the observability of the equation generated by $A$ is known, using the additive perturbation arguments above applied to $U_{1}$ and $U_{2}$ one can derive observability inequalities for the continuous average $\int_{\sigma_{1}}^{\sigma_{2}} u(t, \sigma) d \sigma$. We refer to [17] for a systematic analysis of this kind of situations.

Let us now consider the more general case where the initial data of the abstract system (37) depend on $\sigma$. Consider

$$
\begin{aligned}
u_{t}+\sigma A u & =0, \quad t \in \mathbf{R}^{+} \\
u(0) & =u^{0}(\sigma) .
\end{aligned}
$$


Then

where $U(\cdot, \sigma)$ solves

$$
u(t, \sigma)=U(\sigma t, \sigma),
$$

$$
\begin{aligned}
U_{t}+A U & =0, \quad t \in \mathbf{R}^{+} \\
U(0) & =u^{0}(\sigma) .
\end{aligned}
$$

Obviously, it is easy to see the average of $U(\cdot, \sigma)$ with respect to $\sigma, V=V(t)$, solves the same abstract equation with the average of $u^{0}(\sigma)$ as initial data. But, this does not yield the average of $u(t, \sigma)$ since it corresponds to values of the solution $U(\cdot, \sigma)$ for different values of $t$, depending on $\sigma$.

\subsection{Continuous averages of wave equations}

Let us now consider the case of wave equations with an unknown velocity of propagation parameter $0<\sigma_{1} \leq \sigma \leq \sigma_{2}<\infty$ :

$$
\begin{aligned}
u_{t t}-\sigma^{2} \Delta u & =0, \quad(t, \mathbf{x}) \in \mathbf{R}^{+} \times \Omega \\
u & =0, \quad(t, \mathbf{x}) \in \mathbf{R}^{+} \times \partial \Omega, \\
u(0, \cdot)=u^{0} \in L^{2}(\Omega), & u_{t}(x, 0)=u^{1} \in H^{-1}(\Omega) .
\end{aligned}
$$

As above, we are interested in the problem of determining the initial datum out of measurements done on the average

$$
v(x, t)=\int_{\sigma_{1}}^{\sigma_{2}} u(x, t ; \sigma) d \sigma,
$$

in $L^{2}(\omega \times(0, T))$.

We assume that $\omega$ is an open subset of $\Omega$ satisfying the GCC for the wave equation with the highest velocity of propagation $\sigma_{2}$. We intend to apply the results in [14] that ensure the observability of the additive superposition of two solutions of two wave equations with different propagation speeds.

Proceeding as above we see that

$$
U_{\sigma}(x, t ; \sigma)=u(x, t / \sigma ; \sigma),
$$

solves the same wave equation with $\sigma=1$. Note however that, this time, the initial velocity of $U$ depend on the parameter $\sigma$ so that

$$
U(x, 0 ; \sigma)=U^{0}(x) ; U_{t}(x, 0 ; \sigma)=\frac{1}{\sigma} u^{1}(x) .
$$

So, for the argument of the previous section to be applied directly we rather prepare the initial data in (40) by multiplying the initial velocity by $\sigma$ so that the system reads

$$
\begin{aligned}
u_{t t}-\sigma^{2} \Delta u & =0, \quad(t, \mathbf{x}) \\
u=0, \quad(t, \mathbf{x}) & \in \mathbf{R}^{+} \times \Omega \\
u & \mathbf{R}^{+} \times \partial \Omega, \\
u(0, \cdot)=u^{0} \in L^{2}(\Omega), u_{t}(x, 0)=\sigma u^{1} & \in H^{-1}(\Omega) .
\end{aligned}
$$

This time, the corresponding $U$ is independent of $\sigma$ and satisfies

$$
\begin{aligned}
U_{t t}-\Delta U=0, \quad(t, \mathbf{x}) & \in \mathbf{R}^{+} \times \Omega \\
U=0, \quad(t, \mathbf{x}) & \in \mathbf{R}^{+} \times \partial \Omega, \\
U(0, \cdot)=u^{0} \in L^{2}(\Omega), U_{t}(x, 0)=u^{1} & \in H^{-1}(\Omega) .
\end{aligned}
$$

Then, for system (43),

$$
u(x, t ; \sigma)=U(x, \sigma t)
$$


and therefore

$$
v(x, t)=\int_{\sigma_{1}}^{\sigma_{2}} u(x, t ; \sigma) d \sigma=\int_{\sigma_{1}}^{\sigma_{2}} U(x, \sigma t) d \sigma=\frac{1}{t}\left[V\left(x, \sigma_{2} t\right)-V\left(x, \sigma_{1} t\right)\right],
$$

where

$$
V(x, t)=\int_{0}^{t} U(x, s) d s+\xi(x)
$$

with $\xi(x)$ solution of

$$
\begin{array}{rlrl}
-\Delta \xi & =u^{1}, & & \mathbf{x} \in \Omega \\
\xi & =0, & \mathbf{x} \in \partial \Omega
\end{array}
$$

which is a solution of

$$
\begin{array}{r}
V_{t t}-\Delta V=0, \quad(t, \mathbf{x}) \in \mathbf{R}^{+} \times \Omega \\
V=0, \quad(t, \mathbf{x}) \in \mathbf{R}^{+} \times \partial \Omega, \\
V(0, \cdot)=\xi \in H_{0}^{1}(\Omega), V_{t}(x, 0)=u^{0}(x) \in L^{2}(\Omega) .
\end{array}
$$

Consequently

$$
t v(x, t)=\left[V\left(x, \sigma_{2} t\right)-V\left(x, \sigma_{1} t\right)\right] .
$$

We observe at this level an interesting smoothing effect due to the averaging. Indeed, while each $u(x, t ; \sigma)$ is a weak solution in the space $C\left([0, T] ; L^{2}(\Omega)\right) \cap C^{1}\left([0, T] ; H^{-1}(\Omega)\right)$, the averaged function $t v$, in the presence of the time weight $t$, belongs to $C\left([0, T] ; H_{0}^{1}(\Omega)\right) \cap C^{1}\left([0, T] ; L^{2}(\Omega)\right)$, because the initial data corresponding to $U$ belong to the energy space $H_{0}^{1}(\Omega) \times L^{2}(\Omega)$.

In view of the fact that $(\omega, T)$ satisfies the GCC with the highest velocity of propagation $\sigma_{2}$, according to the results in [14], it turns out that $L^{2}(\omega \times(0, T))$ measurements of $t v(x, t)$ yield $L^{2}(\Omega) \times H^{-1}(\Omega)$ estimates of $\left(\xi, u^{0}\right)$, which is equivalent to an estimate of the original initial data $\left(u^{0}, u^{1}\right)$ in $H^{-1}(\Omega) \times H^{-2}(\Omega)$. Accordingly, we have proved the following:

Theorem 3.4 Let $\omega$ be an open non-empty subset of $\Omega$ and assume that $(\omega, T)$ satisfies the GCC with the fastest velocity of propagation $\sigma_{2}$.

Then, the averages of the solutions of (42) with respect to the unknown velocity of propagation $\sigma$ is such that

$$
\left\|u^{0}\right\|_{H^{-1}(\Omega)}^{2}+\left\|u^{1}\right\|_{H^{-2}(\Omega)}^{2} \leq C\left\|t \int_{\sigma_{1}}^{\sigma_{2}} u(x, t ; \sigma) d \sigma\right\|_{L^{2}(\omega \times(0, T))}^{2} .
$$

Remark 6 - Note that the estimate (47) exhibits a loss of one derivative on the observed norm. This corresponds to the gain of regularity on the average observed above. This fact was not seen in the observability inequality of the averaged heat equation since in this context the natural observability inequality for the heat equation itself exhibits a loss of an infinity number of derivatives as can be seen in the exponentially degenerating weighs of the Fourier expression of the observability inequality.

- Whether one can recover a stronger norm by observing the $L^{2}$-norm of $\int_{\sigma_{1}}^{\sigma_{2}} u(x, t ; \sigma) d \sigma$ instead of $t \int_{\sigma_{1}}^{\sigma_{2}} u(x, t ; \sigma) d \sigma$ is an interesting problem. Note that, in that case, we can not apply directly the results of [14], since the average, without a weight, can not be written as the additive superposition of solutions of wave equations.

Note that the result above applies to the system (42) where the initial data, and in particular the initial velocity, has been adapted and scaled as a function of $\sigma$. Of course, the result above applies to the original system (40) as well, in the particular case where $u^{1} \equiv 0$. Whether a similar result holds when $u^{1} \neq 0$ is an interesting open problem. 


\section{Perspectives}

The results of section 2 raise a number of interesting open problems. Here we briefly mention some of them.

- The method of proof of Section 2 does not apply for systems of PDEs depending on a countable or continuous parameter. Indeed, this would force us to apply an infinite number of PDE operators to the leading equation and would lead to inequalities beyond the Sobolev setting. This is why, in the previous section, we have addressed this issue with other averaging techniques.

- The problem of observation of wave and heat processes is also well understood in the case where the observation is made through the boundary ([19]). But our proof does not apply in that case, since it is fundamentally based on the possibility of applying the PDE operators on the set where the observation is made.

The same can be said about the recent results in [3] on the observation of the heat equation from sets of positive measure.

- The method does not apply in a straightforward manner when the coefficients in the equation are variable, as mentioned above, except for the case where all the operators involved in the equations under consideration are powers of the same elliptic operator, so that they commute.

To exhibit the difficulty one encounters when doing that, let us consider the case in which the density $\rho=\rho(x)$ in the heat equation is variable,

$$
\rho(x) u_{1, t}-\Delta u_{1}=0, \quad(t, \mathbf{x}) \in \mathbf{R}^{+} \times \Omega,
$$

but the wave component is assumed to solve the wave equation with unit coefficients:

$$
\partial_{t}^{2} u_{2}-\Delta u_{2}=0, \quad(t, \mathbf{x}) \in \mathbf{R}^{+} \times \Omega .
$$

Then, applying the wave operator to $u_{1}$ we get that $v_{1}=\left(\partial_{t}^{2}-\Delta\right) u_{1}$ solves

$$
\rho(x) v_{1, t}-\Delta v_{1}-2 \nabla \rho(x) \cdot \nabla u_{1, t}-\Delta \rho(x) u_{1, t}=0 .
$$

Although the extra terms $2 \nabla \rho(x) \cdot \nabla u_{1, t}+\Delta \rho(x) u_{1, t}$ are lower order ones, they are not of local nature in terms of the new unknown $v_{1}$. It is well known that the Carleman inequalities normally employed to derive observability inequalities for heat equations ([12]) are very flexible to handle lower order terms of local nature, but things are much more delicate for non-local ones, because of the lack of commutativity with the exponential weights of the inequality. This is one of the major difficulties when extending the Carleman inequalities from heat equations to Stokes like ones, due to the presence of the pressure term. - In the context of variable coefficients, things could be easier to handle when the main dynamics is given by the wave equation and the heat one is considered as a perturbation. This is so because the techniques based on microlocal defect measures are better adapted to handle lower order terms. But a systematic analysis of this issue remains to be developed.

For instance, in the particular case above, we see that $v_{2}=\left[\rho(x) \partial_{t}-\Delta\right]\left(u_{1}+u_{2}\right)=\left[\rho(x) \partial_{t}-\Delta\right] u_{2}$, solves

$$
\partial_{t}^{2} v_{2}-\Delta v_{2}=2 \nabla \rho(x) \cdot \nabla \partial_{t} u_{2}+\Delta \rho(x) \partial_{t} u_{2}
$$

This equation has to be understood as coupled with the wave equation satisfied by $u_{2}$.

We observe however that the reminder terms of the equation satisfied by $v_{2}$ involve derivatives of $u_{2}$, thus making the issue of the observability of $v_{2}$ delicate and requiring significant further analysis.

Applying the well-known observability inequality for the equation satisfied by $v_{2}$ we obtain that, under the GCC,

$$
\left\|v_{2}^{0}\right\|_{H^{1}}^{2}+\left\|v_{2}^{1}\right\|_{L^{2}}^{2} \leq C \int_{0}^{T} \int_{\omega} v_{2, t}^{2} d x d t+C\left\|u_{2}\right\|_{H^{1}\left(0, T ; H^{1}(\Omega)\right)}^{2} .
$$


Observe that the norm $\left\|v_{2}^{0}\right\|_{H^{1}}^{2}+\left\|v_{2}^{1}\right\|_{L^{2}}^{2}$ is equivalent to that of $\left(u_{2}^{0}, u_{2}^{1}\right)$ in $H^{3} \times H^{2}$. On the other hand, the reminder term involving $\left\|u_{2}\right\|_{H^{1}\left(0, T ; H^{1}(\Omega)\right)}$ can be bounded above by the norm of $\left(u_{2}^{0}, u_{2}^{1}\right)$ in $H^{2} \times H^{1}$. Thus,

$$
\left\|u_{2}^{0}\right\|_{H^{3}}^{2}+\left\|u_{2}^{1}\right\|_{H^{2}}^{2} \leq C \int_{0}^{T} \int_{\omega} v_{2, t}^{2} d x d t+C\left[\left\|u_{2}^{0}\right\|_{H^{2}}^{2}+\left\|u_{2}^{1}\right\|_{H^{1}}^{2}\right] .
$$

Following the classical compactness uniqueness argument one could remove the reminder term to show that

$$
\left\|u_{2}^{0}\right\|_{H^{3}}^{2}+\left\|u_{2}^{1}\right\|_{H^{2}}^{2} \leq C \int_{0}^{T} \int_{\omega} v_{2, t}^{2} d x d t
$$

thus getting the inequality

$$
\left\|u_{2}^{0}\right\|_{H^{3}}^{2}+\left\|u_{2}^{1}\right\|_{H^{2}}^{2} \leq C\left\|u_{1}+u_{2}\right\|_{H^{3}(\omega \times(0, T))}^{2},
$$

but this would require the property of unique continuation ensuring that $v_{2, t} \equiv 0$ in $\omega \times(0, T)$, implies $u_{2}^{0} \equiv u_{2}^{1} \equiv 0$.

Whether this unique continuation property holds or not is an interesting open problem.

The results of Section 3 lead also to very interesting questions. The first one, of course, is to which extent the results presented here in the simplest case where the unknown parameter enters in the system as a simple multiplicative factor of the operator generating the dynamics, can be generalized to larger classes of parameter-dependent PDEs. One could consider, for instance, heat and wave equations involving elliptic operators of the form

$$
-\operatorname{div}(a(x, \sigma) \nabla \cdot) \text {. }
$$

There is some literature addressing specific cases but from the perspective of homogenization or singular perturbations, where the goal is to prove uniform controllability properties with respect to the parameter $\sigma$, i. e. the fact that the equation can be controlled for all values of $\sigma$ and with a cost independent of $\sigma$ (see [19] for the bibliography on that topic.) But nothing is known, as far as we know, when one deals with averages with respect to that parameter.

- The articles [14] and [13] address these issues using microlocal defect measures as a tool to derive observability inequalities for superpositions of PDE of different nature under, among others, the key assumption that the characteristic manifolds do not intersect. In [14] a system of two different wave equations is considered, while in [13] the issue is addressed in more generality, handling, for instance, the superposition of solutions of Schrödinger equations, using parabolic microlocal defect measures.

- The averaged observability of $1 d$ wave and Schrödinger equations, depending continuously on an unknown parameter, has been addressed in [15]. The main tool there is to derive averaged versions of the Ingham inequalities.

\section{Acknowledgements}

The author was supported by the Advanced Grant NUMERIWAVES/FP7-246775 of the European Research Council Executive Agency, FA9550-14-1-0214 of the EOARD-AFOSR, the BERC 2014-2017 program of the Basque Government, the MTM2011-29306, MTM2014-52347 and SEV-2013-0323 Grants of the MINECO, the CIMI-Toulouse Excellence Chair in PDEs, Control and Numerics and a Humboldt Award at the University of Erlangen-Nürnberg.

The author acknowledges Martin Lazar and Qi Lü for interesting discussions. 


\section{References}

[1] F. Ammar-Khodja, A. Benabdallah, M. GonzÁlez-Burgos, L. De Teresa, Recent results on the controllability of linear coupled parabolic problems: a survey, Math. Control Relat. Fields 1(3) (2011) 267-306.

[2] G. Alessandrini and L. Escauriaza, Null-controllability of one-dimensional parabolic equations, ESAIM Control Optim. Calc. Var. 14 (2) (2008) 284293.

[3] J. Apraiz, L. Escauriaza, G. Wang And C. Zhang, Observability inequalities and measurable sets, J. Europ. Math. Soc. 16(11), (2014), 2433-2475.

[4] N. Burq And P. GÉrard, Condition nécessaire et suffisante pour la contrôlabilité exacte des ondes, C. R. Acad. Sci. Paris Sér. I Math., 325(7) (1997) 749-752.

[5] C. Castro and E. Zuazua, Concentration and lack of observability of waves in highly heterogeneous media. Archive Rational Mechanics and Analysis, 164 (1) (2002), 39-72.

[6] M. Choulli, Une introduction aux problèmes inverses elliptiques et paraboliques, Mathématiques \& Applications, 65, Springer, 2009.

[7] S. Chowdhury, D. Mitra, M. Ramaswamy, M. Renardy, Null Controllability of the Linearized Compressible Navier Stokes System in One Dimension, Jl. Differential Equations, 257 (10), (2014), 3813 - 3849.

[8] R. DÁGER AND E. ZuAZUA, Wave propagation and control in $1-d$ vibrating multi-structures. Springer Verlag. "Mathématiques et Applications", vol. 50, 2006.

[9] S. Ervedoza And E. ZuAzuA, Sharp observability estimates for heat equations, Archive for Rational Mechanics and Analysis 202(3) (2011) 975-1017.

[10] S. Ervedoza And E. ZuAzuA, , A systematic method for building smooth controls for smooth data, Discrete Cont. Dyn. Syst. Ser. B, 14(4):1375-1401, 2010.

[11] E. FERnÁndez-CARA AND E. ZuAzuA, The cost of approximate controllability for heat equations: The linear case, Advances in Differential Equations, 5(4-6) (2000) 465-514.

[12] A.V. Fursikov and O.Y. Imanuvilov, Controllability of Evolution Equations, Lecture Notes Series 34, Seoul National University Research Institute of Mathematics, 1996.

[13] M. LAzAr, Stability of observations of Partial Differential Equations under uncertain perturbations, preprint, 2015.

[14] M. Lazar And E. ZuazuA, Averaged control and observation of parameter-depending wave equations, C. R. Acad. Sci. Paris, Ser. I 352 (2014) 497-7502.

[15] J. Loheac And E. ZuazuA, Averaged controllability of parameter dependent one-dimensional wave and Schrödinger equations, preprint, 2015.

[16] A. López AND E. ZuAzUA, Uniform null controllability for the one dimensional heat equation with rapidly oscillating periodic density. Annales IHP. Analyse non linéaire, 19 (5) (2002), 543-580.

[17] Q. Lü AND E. ZuAzuA, Averaged controllability for Random Evolution Partial Differential Equations, preprint, 2014.

[18] M. TuCsnak And G. Weiss, Observation and control for operator semigroups, Birkhäuser Verlag, Basel (2009).

[19] E. Zuazua, Controllability and Observability of Partial Differential Equations: Some results and open problems, in Handbook of Differential Equations: Evolutionary Equations, vol. 3, C. M. Dafermos and E. Feireisl eds., Elsevier Science, 2006, pp. 527-621.

[20] E. ZuazuA, Averaged control, Automatica, 50 (2014) 3077-3087. 